\title{
Protocol
}

\section{A Pathway to Precision Medicine for Aboriginal Australians: A Study Protocol}

\author{
Yeu-Yao Cheng ${ }^{1,2, *} \mathbb{D}^{\text {, Jack Nunn }}{ }^{3}$, John Skinner ${ }^{1}{ }^{\circledR}$, Boe Rambaldini ${ }^{1}$, Tiffany Boughtwood ${ }^{4,5}{ }^{1}$, Tom Calma ${ }^{1}$, \\ Alex Brown ${ }^{6}$, Cliff Meldrum ${ }^{7}$, Marcel E. Dinger ${ }^{8}{ }^{(0}$, Jennifer A. Byrne ${ }^{9,10}{ }^{(0}$, Debbie McCowen ${ }^{11}$, Jayden Potter ${ }^{11}$, \\ Kerry Faires ${ }^{11}$, Sandra Cooper ${ }^{12,13,14}$ and Kylie Gwynne ${ }^{1,15, *(1)}$
}

check for

updates

Citation: Cheng, Y.-Y.; Nunn, J.;

Skinner, J.; Rambaldini, B.;

Boughtwood, T.; Calma, T.; Brown, A.;

Meldrum, C.; Dinger, M.E.; Byrne,

J.A.; et al. A Pathway to Precision

Medicine for Aboriginal Australians:

A Study Protocol. Methods Protoc.

2021, 4, 42. https://doi.org/

$10.3390 / \mathrm{mps} 4020042$

Academic Editor: Fernando Albericio

Received: 22 May 2021

Accepted: 15 June 2021

Published: 21 June 2021

Publisher's Note: MDPI stays neutral with regard to jurisdictional claims in published maps and institutional affiliations.

Copyright: (c) 2021 by the authors. Licensee MDPI, Basel, Switzerland. This article is an open access article distributed under the terms and conditions of the Creative Commons Attribution (CC BY) license (https:// creativecommons.org/licenses/by/ $4.0 /)$.
1 Poche Centre for Indigenous Health, The University of Sydney, Camperdown, NSW 2050, Australia; john.skinner@sydney.edu.au (J.S.); boe.rambaldini@sydney.edu.au (B.R.); tom.calma@sydney.edu.au (T.C.)

2 Faculty of Medicine and Health, The University of Sydney School of Medicine, Camperdown, NSW 2050, Australia

3 School of Psychology and Public Health, La Trobe University, Melbourne, VIC 3086, Australia; jack.nunn@latrobe.edu.au

4 Australian Genomics Health Alliance, Melbourne, VIC 3052, Australia; tiffany.boughtwood@mcri.edu.au

5 Murdoch Children's Research Institute, Melbourne, VIC 3052, Australia

6 South Australian Health and Medical Research Institute, Adelaide, SA 5000, Australia; alex.brown@sahmri.com

7 NSW Health Pathology, Sydney, NSW 2065, Australia; cliff.meldrum1@health.nsw.gov.au

8 School of Biotechnology and Biomolecular Sciences, UNSW, Sydney, NSW 2052, Australia; m.dinger@unsw.edu.au

9 New South Wales Health Statewide Biobank, New South Wales Health Pathology, Camperdown, NSW 2050, Australia; jennifer.byrne@health.nsw.gov.au

10 School of Medical Sciences, Faulty of Medicine and Health, The University of Sydney, Camperdown, NSW 2006, Australia

11 Armajun Aboriginal Health Service, 1 Rivers Street, Inverell, NSW 2360, Australia; dmccowen@armajun.org.au (D.M.); potsy2009@gmail.com (J.P.); admin@armajun.org.au (K.F.)

12 Kids Neuroscience Centre, Children's Hospital at Westmead, Westmead, NSW 2145, Australia; sandra.cooper@sydney.edu.au

13 Discipline of Child and Adolescent Health, The University of Sydney, Camperdown, NSW 2006, Australia

14 The Children's Medical Research Institute, Westmead, NSW 2145, Australia

15 Faculty of Medicine and Health Sciences, Macquarie University, Sydney, NSW 2113, Australia

* Correspondence: yche6491@uni.sydney.edu.au (Y.-Y.C.); kylie.gwynne@mq.edu.au (K.G.)

Abstract: (1) Background: Genomic precision medicine (PM) utilises people's genomic data to inform the delivery of preventive and therapeutic health care. PM has not been well-established for use with people of Aboriginal and Torres Strait Islander ancestry due to the paucity of genomic data from these communities. We report the development of a new protocol using co-design methods to enhance the potential use of PM for Aboriginal Australians. (2) Methods: This iterative qualitative study consists of five main phases. Phase-I will ensure appropriate governance of the project and establishment of a Project Advisory Committee. Following an initial consultation with the Aboriginal community, Phase-II will invite community members to participate in co-design workshops. In Phase-III, the Chief Investigators will participate in co-design workshops and document generated ideas. The notes shall be analysed thematically in Phase-IV with Aboriginal community representatives, and the summary will be disseminated to the communities. In Phase- $\mathrm{V}$, we will evaluate the co-design process and adapt our protocol for the use in partnership with other communities. (3) Discussion: This study protocol represents a crucial first step to ensure that PM research is relevant and acceptable to Aboriginal Australians. Without fair access to PM, the gap in health outcome between Aboriginal and non-Aboriginal Australians will continue to widen.

Keywords: co-design; precision medicine; personalised medicine; Aboriginal health; participatory research; genomics; Aboriginal and Torres Strait Islanders; Australian 


\section{Introduction}

Genomic precision medicine (PM) leverages established understanding about genomic variation associated with wellness and disease to tailor medical treatment for an individual through targeted analyses of medically relevant information within an individual's sequenced DNA. PM can positively impact all stages of clinical management, improving diagnosis, pinpointing appropriate preventive measures, and enabling therapeutic interventions [1,2]. To make PM more effective, genomic researchers group DNA sequence information from diverse global sub-populations, such as shared ancestry groupings, as people within these groupings will have a greater proportion of shared DNA traits [3]. These groups can affect the nature and direction of the research undertaken and the interventions that are available to those groups.

While PM is already being used worldwide to improve lives, its utility and effectiveness is not maximised for individuals with Aboriginal and Torres Strait Islander ancestry as there is less genomic data from people within these groupings $[4,5]$. There are no current population-specific data for Aboriginal Australians curated within the Genome Aggregation Database [6,7]. The deficit affects equity of access to PM for Aboriginal people, who represent a culturally and linguistically diverse set of communities at greater risks of poorer health outcomes $[3,8]$. In the era of rapidly advancing medical care and technology, there is a serious risk that this inequity will widen the already prominent health and life expectancy gaps between Aboriginal and non-Aboriginal populations $[9,10]$. The Australian government and state governments have developed health genomics plans and policy frameworks that include various strategies to increase the use of PM for Aboriginal Australians [11].

Genomic research is particularly sensitive and complex because genetics has been used in the past to justify and perpetuate racial discrimination [12-14]. Past research practice has positioned Indigenous peoples as research subjects rather than equal partners in the research process [15]. Historically, the lack of study transparency and research malpractice has also undermined trust in many scientific investigations and heightened concerns among Indigenous peoples about sharing personal health information [12]. While some people may have concerns regarding genetic research, such as genetic discrimination in employment, difficulty in obtaining insurance, and inappropriate use of genomic data in law enforcement (e.g., fabricated DNA evidence in crime scenes) [14], Indigenous peoples may have additional concerns specific to their cultural, social, and collective contexts. This may include allegations of genetic inferiority, threats to cultural beliefs, fear of exploitation for commercial purposes (e.g., drug development), and inappropriate use in Native Title claims or exclusion from government assistance [13]. These concerns highlight the importance of adopting a collaborative approach to conducting genomic research involving Indigenous peoples, one that partners with Indigenous communities and incorporates Indigenous perspectives and culture into study design and conduct.

Participatory Action Research (PAR) is an umbrella term for study models such as "co-creation" or "co-design", which embraces a participatory philosophy [16]. Placing impacted communities at the heart of the process, the benefits of co-design include: the empowerment of vulnerable communities, better identification of priority topics relevant to the communities, generation of innovative ideas, reduction of scientific bias, and the potential for long-term collaboration on future projects $[17,18]$. In co-design, Aboriginal communities are involved in every step of the study: the development of the research question, design of the study methodology, determining governance of data, and the interpretation and dissemination of results. These stages of the study are continuously evaluated and refined to reflect the interest and values of the participating communities and co-design partners. "Involvement" is when power is shared with the research participants to actively contribute to the process of research [19] and is distinct from "engagement" where only information and knowledge is shared [20].

Crucially, the purpose of co-design is not limited to increasing the participation of Aboriginal people in genomic research but extends to improving research and service 
design as a target in its own right. Previous studies have shown that even when health services are available, desired health outcomes are not achieved if they are not designed for and with Aboriginal people [21]. Aboriginal health workforce development strategies (e.g., training of Aboriginal genetic counsellors and pathology collection professionals) should also be developed in parallel to deliver health care in a culturally safe manner. Aboriginal workforce training will not only support greater participation in, and benefits realised from, genomic PM - but those trained will also be able to meet other medical needs for their communities.

PM may play a role in closing the health gap for Aboriginal Australians, and will rely on co-design to enable the development and appropriateness of such health services [22,23]. In this paper, we propose a protocol for community involvement and co-design to collect aggregated genomic data.

\section{Study Design}

The current study is an iterative qualitative study that aims to begin the co-design process with three different Aboriginal communities. We bring together experts in five important areas: Aboriginal culture, community engagement, research co-design, genomics, and public policy. As shown in Figure 1 and described in greater detail later, the study consists of five main phases: Preparation, Community Involvement, Data Collection, Data Analysis and Dissemination of Results, and Adaptation.
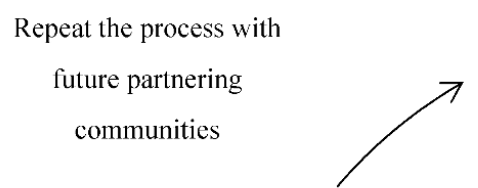

\section{Phase V: Adaptation}

-Transfer of data

-Revision of PM protocol

-Evaluation of the co-design process
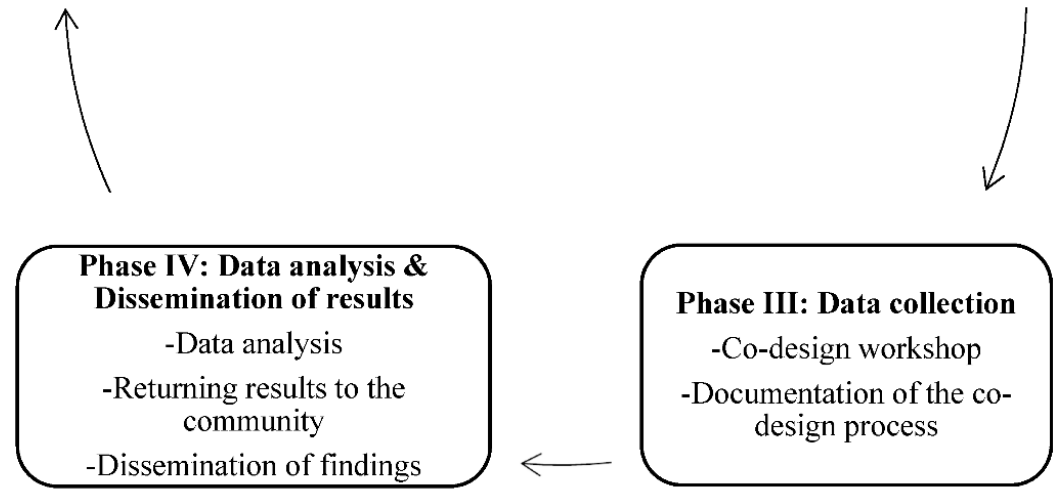

Figure 1. Five Phases of the Co-design Protocol.

While the overall study structure is similar for all communities involved, the specific details of the study within each respective community could vary considerably owing to the co-design process. For example, there may be changes to the language, images, and analogies used to explain PM, and there may be different arrangements with respect to management and storage of samples and data. Furthermore, during the Adaptation phase, 
ideas generated with the first community will help shape future co-design workshops with subsequent communities, highlighting the strength and iterative nature of Participatory Action Research. It is predicted that the process of adapting the protocol will take three months for each new community.

\section{Methods}

\subsection{Phase I: Preparation}

\subsubsection{Ensuring Aboriginal Governance}

Previous studies have demonstrated that research with Aboriginal people that is led by Aboriginal researchers is more culturally relevant, effective, and readily adopted [24]. Our core research team of 10 researchers includes five Aboriginal Chief Investigators (CI) who have substantial research experience. The CI's will be responsible for the overarching guidance, design, and conduct of the entire project.

In addition to the core research team, an independent Project Advisory Committee (PAC) [25] will be established and jointly chaired by an Aboriginal Elder nominated by the community and the director of the Poche Centre for Indigenous Health of the University of Sydney who is an Aboriginal Elder and senior researcher. Each community will have its own PAC and will meet bi-monthly. The primary role of the PAC will be to ensure Aboriginal control, governance, and timely completion of the project. The PAC will oversee the cultural safety and integrity of the project, as well as advise on the dissemination and sharing of results to influence policy and practice based on research findings. An annual progress report will be provided to the Aboriginal Health and Medical Research Council (AHMRC) Ethics Committee, which reviews all presentations and publications prior to presentation and submission. The chairs of the different PACs will have the opportunity to communicate, which allows for the consideration of feedback and experiences from communities other than their own.

\subsubsection{Developing Research Team Cultural Competency}

There are currently no specific Australian guidelines for cultural competency training, requirements, or standards for researchers involved in Aboriginal genetic studies [26]. We will follow the guidelines from National Health and Medical Research Council (NHMRC) [27] and strategies outlined in Genomic Partnerships [28] to engage and partner with Aboriginal peoples for genomic research. All investigators will either have extensive experience working with Aboriginal communities or will complete cultural competence training [29] and pre-readings on selected Aboriginal genetic research articles. Investigators will also familiarize themselves with the recommendations of the Aboriginal Knowledge and Intellectual Property Protocol [30]. All investigators will be expected to interact with Aboriginal communities with respect, humility, empathy, and willingness to listen throughout all the stages of the study. Feedback about Investigators and their behaviours when working with Aboriginal people and communities will be managed through each PAC.

\subsubsection{Initial Community Consultation to Explore Views of Impacted Communities}

In partnership with Armajun Aboriginal Health Service (AHS), the Poche Centre has developed a plain English summary of the PM project. A video created by the National Centre for Indigenous Genomics at the Australia National University [31] has been adapted to explain genomic precision medicine visually and in plain English. Armajun AHS proposed an Aboriginal community in New South Wales (NSW) for initial community involvement because they had some experience and health literacy with genetic medicine. In addition, our research team had been collaborating with Armajun AHS for more than five years on several co-design research projects in oral health, cardiovascular disease, and lung health. This research was valued by the community and the research team. The introductory video [32] and information sheets were made widely available in the community. An online survey [33] was conducted by the Armajun AHS and the Local Aboriginal Land Council (LALC) to determine community support for the project. An 
informal discussion was used to explore people's views and preferences for involvement. Members of the community also helped to improve the dissemination plan for letting more people in the community know about the planned study.

An interviewer assisted online anonymous survey was conducted via SurveyMonkey (Supplement 1) [34] by the Aboriginal Community Controlled Health Services (ACCHS) and the LALC. The survey consisted of eight questions gathering the individual's basic demographic information (age, Indigenous status, geographical location), thoughts on the PM video, and support (or the lack thereof) for the study. Through the consultation, it was agreed that at least $10 \%$ Aboriginal community support was required for the LALC and Armajun AHS to provide letters of support for the human research ethics application. The survey indicated a high level of support and willingness to co-design, with $100 \%$ ( $n=51)$ community members surveyed supporting the study in less than three weeks. Of the 51 participants who watched the video and responded to the survey ( $47 \%$ male, $78 \%$ younger than $55 \mathrm{y}), 19(42 \%)$ shared their thoughts on the project. Participants suggested face-to-face events as the preferred method for co-design. One-hundred percent $(n=51)$ of respondents believed it was a good idea to collect DNA samples from the community to form the DNA library. Letters of support were subsequently provided from the ACCHS and the LALC (Supplement 2).

\subsection{Phase II: Community Involvement}

\subsubsection{Selection of Aboriginal Communities}

Where consent is granted by the local AHS following the Preparation phase, we will invite prospective communities to participate in co-design. We will follow the actions of the dissemination plan developed with community members during the "initial community consultation" of the Preparation phase. This includes engaging in mainstream print, TV media, social platforms, and advertisements in public places such as shop windows. The co-design workshops will be open to all members of the communities. Local AHSs will also be asked to nominate suitable prospective participants. Where possible, we will ensure that the group is heterogenous as we seek diverse experiences and opinions related to genetic testing.

\subsubsection{Inclusion and Exclusion Criteria}

People who identify as Aboriginal aged 18 years and above will be eligible to participate in the co-design workshop that will take place at the LALC and ACCHS.

\subsubsection{Consent to Co-Design}

Each participant will be provided a written participant information statement and consent form developed by the AHMRC (Supplement 3) [35]. Participants will be reminded that their participation is entirely voluntary, information gathered during surveys and discussions are anonymous, and withdrawal from the study is allowed at any point without any consequences or implications on their future access to healthcare.

\subsection{Phase III: Data Collection}

\subsubsection{Structure of the Co-Design Workshop}

An Aboriginal Elder will open the workshop with local customs and traditions specific to their community. The opening will be followed by an introduction by the director of the Poche Centre for Indigenous Health about the research institution and staff members present. A 4-min introductory video explaining PM [32] will be played and plain English information sheets will be handed to attendees. The video begins with the Acknowledgement of Country, followed by the explanation of the concept of DNA. The video uses analogies, which illustrate DNA as stories in the blood written in languages, that can be understood by clinicians and scientists to discover why one person gets sick. The video describes DNA as "the story is passed down from our ancestors and that people can share stories with others in the same geographical area even if they are not from the same family." The video 
highlights that the DNA stories allow doctors to understand why some communities are more prone to certain genetic disorders or why certain treatment might work in some family members but not others. Finally, the video states the aim of the project: to create a baseline DNA story so that Aboriginal people can access PM.

Participants will be encouraged to ask questions and provide thoughts on the video and the study. The CIs present will ask open-ended questions, including exploring priority topics relevant to precision medicine. Apart from helping to spark ideas and discussion, the CI's will also be required to ensure an optimal dynamic for the exchange of opinion. The CI's will follow the verbal and non-verbal cues of senior Aboriginal participants in the discussion [36]. The CI's will routinely ask questions such as "is this what you had in mind?" to confirm that the interpretation of the idea is congruent with that of the participants. The "NSW Health Statewide Biobank Consent Toolkit" [37] and the "Patient Information Booklet: Genetic and Genomic Testing" [38] provide standards and guidance for biobanking and consent procedures. We will review these documents along with those from other states to ensure key themes related to PM are co-designed with communities during the workshop. Areas requiring co-design include participant recruitment, consenting, DNA sample collection and storage, data governance and sovereignty, and the dissemination of results and incidental findings. Additionally, workforce involvement (training of local Aboriginal people as genetic counsellors and pathology collectors) will be discussed with the participants. This leverages previous work by the research team, which identified the urgent need to address the insufficient supply of Aboriginal health professionals [39], and Poche Centre's ongoing mission to build and support education and career pathways for Aboriginal people [40].

Although participants will be encouraged during the workshop to voice their thoughts or ask questions, we respect that not all participants are comfortable sharing ideas in front of the larger group. Smaller group discussions will also be facilitated, with the groups reporting feedback both verbally and in writing. Alternative methods of providing feedback and asking questions will be provided during the meeting, including written notes, which can be submitted anonymously if the participant chooses. Regular breaks will ensure that participants can approach members of the research team to discuss ideas or concerns privately if preferred. At the end of the workshop, contact information for the research team will be provided to the participants.

\subsubsection{Documentation the Co-Design Process}

Discussions during the co-design workshop will be summarised with pseudonyms assigned to participants. The note taker will verbally repeat the notes taken and check with participants for accuracy and to ask if they wish to add anything. Locations, communities, and participants will be de-identified to protect confidentiality and privacy. In addition, notes will be shared after the event, with participants given an opportunity to provide further feedback on the notes in writing. The co-design process will also be reported using the STARDIT reporting tool, with a report co-created with the community [41]. The report will contain data about who was involved, how, and in which tasks. Information about the co-design method, the data analysis methods, reported enablers and barriers for involvement, and any other impacts and outcomes from the co-design process will also be reported.

\subsection{Phase IV: Data Analysis \& Dissemination of Results}

\subsubsection{Data Analysis}

Quantitative data gathered through surveys regarding the group's demographic makeup will be analysed through descriptive statistics. For the qualitative data, we will perform an inductive thematic analysis using NVivo to organize participant's responses into key themes. Coding and thematic analysis of qualitative data will be carried out by two members of the study team and checked by another author, following best practices for enhancing validity in qualitative methods $[42,43]$. At least one of the three investigators involved in coding and analysis will be Aboriginal. The core research team will meet to re- 
view the findings and identify outstanding or representative quotes for future presentation of the results. Preliminary findings will be discussed with each PAC.

\subsubsection{Returning Results to the Community}

Results of the co-design study and a draft protocol for the PM study will be disseminated into the local Aboriginal community through community meetings and printed research summaries (including Plain English summaries). We will work in collaboration with ACCHS to hold community meetings and information evenings, which both participants and non-participants of the study can attend. Community members will be encouraged to provide feedback and comments on the draft local protocol and modified introductory video and information sheet to ensure that our interpretation is consistent with the views of the community and co-design participants.

Results will be published in peer reviewed journals and presented at professional conferences. ACCHSs participating in the study will be invited to contribute to these publications and presentations. We will acknowledge the sources of information and those who have contributed to the research through authorship and acknowledgement in resulting publications, meetings with community members and conference presentations. We will also acknowledge the cultural property rights of Aboriginal peoples in relation to knowledge, ideas, cultural expressions, and cultural materials by including AHS representatives as Chief Investigators on the study.

\subsubsection{Dissemination of Results}

An integrated knowledge-to-action (KTA) plan will be developed to detail our engagement of key stakeholders in the research process and disseminate ongoing findings. The types of KTA activities will be determined in collaboration with our project partners, co-design participants, and the PAC. The diversity of stakeholders and organisations involved our project will ensure that our research findings inform policy and practice, leading to the development of personalised medicine. Our KTA strategies will include multiple strategies such as social media (e.g., Facebook, Twitter, Instagram, and LinkedIn), mass media communications, policy papers, conference presentations and open access journal publications, short video and podcasts, and public events (e.g., barbecues, picnics, and campfires).

\subsection{Phase V: Adaptation}

\subsubsection{Transference of Data from the Co-Design Team to the Precision Medicine Team}

The PM project consists of three stages: the co-design study, workforce involvement, and biobanking. The co-design study serves to create a framework and inform the subsequent stages of the PM project. All investigators will have access to findings of the co-design study (e.g., stakeholder's view on appropriate biobanking and consenting). Further details on data management of the latter stages of the PM project (e.g., DNA samples, DNA sequences) will be co-designed with the communities. The processes of data transference used in the project will be consistent with the principles of Participatory Action Research where stakeholders collectively decide upon roles, responsibilities, and data access [23].

\subsubsection{Revising and Refining the Precision Medicine Project}

Supplement 4 is a plain English summary of the most up-to-date version of the PM project, which will be revised based on findings of the current participatory study. Following the conclusion of this study, investigators will meet to discuss how to refine the PM project with input from the PAC. Elements of the PM project include the following: the consent process, collection and storage of samples, governance of data, and reporting findings will be reconstructed to align with the views of participants and stakeholders.

\subsubsection{Reporting and Evaluating Co-Design}

To date, there are no guidelines on how to objectively assess the quality of co-design processes. However, New Philanthropy Capital (NPC) have suggested that co-design can be 
assessed based on three areas: (1) benefits to the participants, (2) the quality of the process, and (3) the insights obtained to achieve the intended outcome [44]. Although the discussion of an objective evaluation of co-design is beyond the scope of this paper, the authors appreciate the importance, challenges, and potential ways of evaluating co-design, and they will be selectively using a combination of resources to appraise and uphold the integrity and quality of our work. We propose the use of Standardised Data on Initiatives (STARDIT) [41] to report, evaluate, and keep public records of every stage of the co-design process.

\section{Discussion}

To make precision medicine available to Aboriginal and Torres Strait Islanders, the broader PM project uses the Five Aim Approach (Figure 2): (1) Protocol co-design, (2) Capacity building (training of Aboriginal professionals for genetic counselling and pathology collection), (3) Culturally safe sample collection, (4) Data analysis \& bioinformatics, and (5) Translation. The manuscript is the protocol paper for the first part (Protocol codesign) of the broader PM study, with a focus on creating a methodology for community engagement and involvement that is feasible and acceptable. It represents a critical first step to improve and enable PM related health service delivery and to close the health gap between Indigenous and non-Indigenous populations. While co-design is the first part of PM project, co-production is continuous throughout the entire PM project, with Aboriginal communities, CIs, and the PAC involved as co-researchers for all the stages of the project.

The value of Participatory Action Research with Indigenous communities is well documented $[17,18,22,23]$. The concept of "involvement" gives rise to the main strengths of the study: fluidity, adaptability, and cultural safety. As the nature of our topic (i.e., genomics research and research with Aboriginal communities) is highly complex and sensitive, co-design becomes not only preferred but necessary due to its emphasis on both the process and result of research. Although time consuming at the initial stages, by involving participants early on, we ensure that the study is feasible, the topic areas are relevant and meet stakeholder needs, and the study process upholds the required cultural respect and integrity. In the long-run, using a co-design process saves time, promotes the usage of health services, and elicits superior health outcomes [21]. Other benefits specific to our PM project that support the use of co-design include the building of strong and committed community partners and enhancing skills and knowledge in the Aboriginal community about genomics and health.

By prioritising equity and valuing culture and community over research outputs, the current paper delivers the necessary guiding framework (culturally acceptable recruitment of participants, informed consent, and governance of data and samples) to answer important questions about data ethics, security, and quality associated with genomic research. We bring together experts from various disciplines to collaboratively develop the protocol according to published guidelines on ethical engagement of Aboriginal people in genomic research [18] and previously identified enablers for effective health service delivery for Aboriginal Australians [21]. The next step for the study is to apply for ethics approval from the AHMRC, after which we will commence with community involvement.

\section{Limitations}

The protocol shares limitations that are present in many other Participatory Action Research studies. This includes not being able to elucidate the precise details (e.g., content of the co-design workshop) at the time of writing this study protocol and the lack of standardised ways to report co-design. Despite these limitations, a major benefit of codesign is the discovery of relevant topics, enablers, and barriers that are not yet known to the research team. At this point, we provide a general direction for co-design topics that is relevant to all genomic studies, which will be refined by participants. We will transparently document and evaluate the co-design process. 

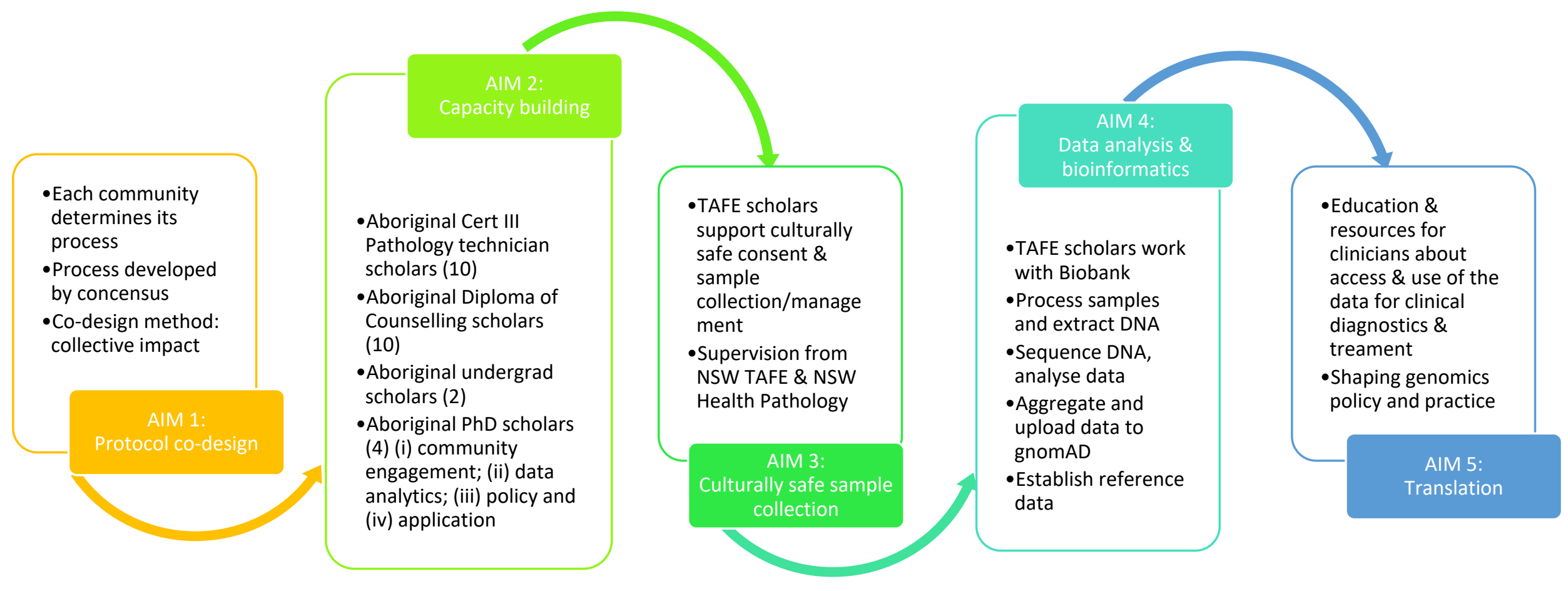

Figure 2. Five aim approach to enable Aboriginal Precision Medicine. 
Supplementary Materials: The following are available online at https:/ /www.mdpi.com/article/10 $.3390 / \mathrm{mps} 4020042 / \mathrm{s} 1$.

Author Contributions: Conceptualization, Y.-Y.C., J.N., J.S., T.B. and K.G.; methodology, Y.-Y.C., J.N., J.S., T.B. and K.G.; formal analysis, Y.-Y.C., J.N., J.S., T.B., S.C., B.R. and K.G.; writing-original draft preparation, Y.-Y.C., J.N., J.S. and K.G.; writing-review and editing, Y.-Y.C., J.S., B.R., T.B., J.A.B., C.M., D.M., J.P., K.F., S.C., M.E.D., A.B., T.C. and K.G. All authors have read and agreed to the published version of the manuscript.

Funding: Alex Brown is supported by the NHMRC Fellowship (GNT1137563) and The Hospital Research Foundation (SA). Kylie Gwynne is supported by Australian Genomics for work associated with Mackenzie's mission. Australian Genomics is supported by the NHMRC (GNT1113531, GNT2000001) and the Australian Government's Medical Research Futures Fund. The current protocol has not received any external funding. Stated above are fundings for individual investigators for related work.

Institutional Review Board Statement: The Aboriginal Health \& Medical Research Council Human Research Committee granted ethics approval to the study on 31 March 2021. The project was deemed to meet the requirements of the National Statement on Ethical Conduct in Human Research.

Informed Consent Statement: Informed consent will be obtained from all subjects involved in the study. At the time of writing this protocol paper, participant recruitment has not yet commenced.

Data Availability Statement: The datasets used during the current study are available from the corresponding author on reasonable request.

Acknowledgments: We would like to acknowledge Armajun Aboriginal Health Service and the Local Aboriginal Land Council of the initial community. The work conducted forms part of Y.-Y.C.'s medical degree as a student at the University of Sydney School of Medicine.

Conflicts of Interest: Sandra Cooper is director of Frontier Genomics Pty Ltd. (Sydney, NSW, Australia). Frontier Genomics has not traded (as of July 2020). Frontier Genomics Pty Ltd. (Sydney, NSW, Australia) has no existing financial relationships that will benefit from publication of these data. The remaining co-authors do not have any relationships, financial or otherwise, that may result in a perceived conflict of interest.

\section{References}

1. Rae, K.M.; Grimson, S.; Pringle, K.G. Personalised Medicine: A New Approach to Improving Health in Indigenous Australian Populations. Public Health Genom. 2017, 20, 58-62. [CrossRef]

2. National Research Council (US). Committee on A Framework for Developing a New Taxonomy of Disease. In Toward Precision Medicine: Building a Knowledge Network for Biomedical Research and a New Taxonomy of Disease; National Research Council (US): Washington, DC, USA, 2011.

3. Robertson, S.P.; Hindmarsh, J.H.; Berry, S.; Cameron, V.A.; Cox, M.P.; Dewes, O.; Doughty, R.N.; Gray, G.; Jacobsen, J.C.; Laurence, A.; et al. Genomic medicine must reduce, not compound, health inequities: The case for hauora-enhancing genomic resources for New Zealand. N. Z. Med. J. 2018, 131, 81-89.

4. $\quad$ Easteal, S.; Arkell, R.M.; Balboa, R.F.; Bellingham, S.A.; Brown, A.D.; Calma, T.; Cook, M.C.; Davis, M.; Dawkins, H.J.; Dinger, M.E.; et al. Equitable expanded carrier screening needs Indigenous clinical and population genomic data. Am. J. Hum. Genet. 2020, 107, 175-182. [CrossRef]

5. Bilkey, G.A.; Burns, B.L.; Coles, E.P.; Bowman, F.L.; Beilby, J.P.; Pachter, N.S.; Baynam, G.; Dawkins, H.J.S.; Nowak, K.J.; Weeramanthri, T.S. Genomic Testing for Human Health and Disease Across the Life Cycle: Applications and Ethical, Legal, and Social Challenges. Front. Public Health 2019, 7, 40. [CrossRef]

6. Genome Aggregation Database. Available online: https:/ / gnomad.broadinstitute.org/ (accessed on 26 March 2020).

7. Nowak, K.J.; Bauskis, A.; Dawkins, H.J.; Baynam, G. Incidental inequity. Eur. J. Hum. Genet. 2018, 26, 616-617. [CrossRef] [PubMed]

8. Petrovski, S.; Goldstein, D.B. Unequal representation of genetic variation across ancestry groups creates healthcare inequality in the application of precision medicine. Genome Biol. 2016, 17, 157. [CrossRef] [PubMed]

9. Nunn, J. Reducing health inequalities by involving indigenous people in genomics research. J. Consum. Health Forum Aust. 2017.

10. Holland, C. Close of Gap Progress and Priorities Report 2016; The Close the Gap Campaign Steering Committee: Sydney, Australia, 2016.

11. Commonwealth of Australia Department of Health. Implementation Plan: National Health Genomics Policy Framework 2018-2021; Commonwealth: Sydney, Australia, 2018. 
12. Dukepoo, F.C. Genetic services in the new era: Native American perspectives. Community Genet. 1998, 1, 130-133. [CrossRef] [PubMed]

13. Kowal, E.; Pearson, G.; Rouhani, L.; Peacock, C.S.; Jamieson, S.E.; Blackwell, J.M. Genetic research and Aboriginal and Torres Strait Islander Australians. J. Bioethical Inq. 2012, 9, 419-432. [CrossRef] [PubMed]

14. Middleton, A.; Milne, R.; Thorogood, A.; Kleiderman, E.; Niemiec, E.; Prainsack, B.; Farley, L.; Bevan, P.; Steed, C.; Smith, J.; et al. Attitudes of publics who are unwilling to donate DNA data for research. Eur. J. Med Genet. 2019, 62, 316-323. [CrossRef] [PubMed]

15. Hickey, G.; Brearley, S.; Coldham, T.; Denegri, S.; Green, G.; Staniszewska, S.; Tembo, D.; Torok, K.; Turner, K. Guidance on Co-Producing a Research Project; INVOLVE: Southampton, UK, 2018.

16. Macaulay, A.C. Participatory research: What is the history? Has the purpose changed? Fam. Pract. 2017, 34, 256-258. [CrossRef]

17. Batalden, M.; Batalden, P.; Margolis, P.; Seid, M.; Armstrong, G.; Opipari-Arrigan, L.; Hartung, H. Coproduction of healthcare service. BMJ Qual. Saf. 2016, 25, 509-517. [CrossRef]

18. Claw, K.G.; Anderson, M.Z.; Begay, R.L.; Tsosie, K.S.; Fox, K.; Garrison, N.A. A framework for enhancing ethical genomic research with Indigenous communities. Nat. Commun. 2018, 9, 2957. [CrossRef] [PubMed]

19. National Institute for Health Research. Patient and Public Involvement in Health and Social Care Research. 2015. Available online: https: / / www.rds-yh.nihr.ac.uk/wp-content/uploads/2015/01/RDS_PPI-Handbook_2014-v8-FINAL-11.pdf (accessed on 26 March 2020).

20. Health Research Authority. Public Involvement. Available online: https://www.hra.nhs.uk/planning-and-improving-research/ best-practice/public-involvement/ (accessed on 26 March 2020).

21. Gwynne, K.; Jeffries, T.; Lincoln, M. Improving the efficacy of healthcare services for Aboriginal Australians. Aust. Health Rev. 2018, 43, 314-322. [CrossRef]

22. Goodyear-Smith, F.; Jackson, C.; Greenhalgh, T. Co-design and implementation research: Challenges and solutions for ethics committees. BMC Med Ethics 2015, 16, 78. [CrossRef] [PubMed]

23. Nunn, J.S.; Tiller, J.; Fransquet, P.; Lacaze, P. Public Involvement in Global Genomics Research: A Scoping Review. Front. Public Health 2019, 7, 79. [CrossRef]

24. Clapham, K. Indigenous-led intervention research: The benefits, challenges and opportunities. Int. J. Crit. Indig. Stud. 2011, 4. [CrossRef]

25. National Health and Medical Research Council; Canberra, A.N. Road Map II: A Strategic Framework for Improving the Health of Aboriginal and Torres Strait Islander People Through Research; NHMRC: Canberra, Australia, 2010.

26. Garrison, N.A.; Hudson, M.; Ballantyne, L.L.; Garba, I.; Martinez, A.; Taualii, M.; Arbour, L.; Caron, N.R.; Rainie, S.C. Genomic Research Through an Indigenous Lens: Understanding the Expectations. Annu. Rev. Genom. Hum. Genet. 2019, $20,495-517$. [CrossRef]

27. National Health and Medical Research Council. Ethical Guidelines for Resaerch with Aboriginal and Torres Strait Islander Peoples; Commonwealth of Australia: Canberra, Australia, 2018.

28. QIMR Berghofer. Genomic Partnerships: Guidelines for Genomic Research with Aboriginal and Torres Strait Islander Peoples of Queensland. 2019. Available online: https:/ /www.qimrberghofer.edu.au/wp-content/uploads/2020/09/2019-IndigenousHealth-Genomics-Guide-v9-WEB.pdf (accessed on 26 March 2020).

29. Russel-Mundine, G. Cultural Competence-Aboriginal Sydney. Available online: https://www.coursera.org/learn/culturalcompetence-aboriginal-sydney\#about (accessed on 10 March 2020).

30. Orr, M.K.P.; Gorey, I.N.; Dixon, T.; Mir, A.; Cox, E.; Wilson, J. Aboriginal Knowledge and Intellectual Property Protocol: Community Guide, 2nd ed.; Ninti One Limited: Alice Springs, Australia, 2009.

31. Australian National University. National Centre for Indigenous Genomics; The Australian National University: Canberra, Australia, 2021.

32. The Poche Centre for Indigenous Health. Precision Medicine Community Video. Available online: https://www.youtube.com/ watch?v=S1dsAHONDBw\&feature=youtu.be (accessed on 26 March 2020).

33. The Poche Centre for Indigenous Health. Personalised Medicine Survey. Available online: https://www.surveymonkey.com/r/ JDW9G79 (accessed on 26 March 2020).

34. SurveyMonkey Inc. Available online: www.surveymonkey.com (accessed on 26 March 2020).

35. Aboriginal Health and Medical Counil Ethics Committee. AH\&MRC Ethics Committee Model Consent Form-Individual Participant. Available online: https:/ / www.ahmrc.org.au/publication/ahmrc-ethics-committee-model-consent-form-individualparticipant/ (accessed on 26 March 2020).

36. Point of Care Foundation. EBCD: Experience-Based Co-Design Toolkit. Available online: https://www.pointofcarefoundation. org.uk/resource/experience-based-co-design-ebcd-toolkit/ (accessed on 15 April 2020).

37. NSW Ministry of Health. NSW Health Statewide Biobank Consent Toolkit. 2018. Available online: https://biobank.health.nsw. gov.au/researchers/nsw-health-consent-toolkit (accessed on 26 March 2020).

38. Agency for Clinical Innovation. Patient Information Booklet: Genetic and Genomic Testing. 2017. Available online: https: / / www.aci.health.nsw.gov.au/_data/assets/pdf_file/0010/386830/Consent-Booklet_Genetic-Genomic-Testing.pdf (accessed on 26 March 2020). 
39. Gwynne, K.; Lincoln, M. Developing the rural health workforce to improve Australian Aboriginal and Torres Strait Islander health outcomes: A systematic review. Aust. Health Rev. 2017, 41, 234-238. [CrossRef]

40. The Poche Centre for Indigenous Health. Available online: https://www.sydney.edu.au/medicine-health/our-research/researchcentres/the-poche-centre-for-indigenous-health.html (accessed on 26 March 2020).

41. Nunn, J.S.; Shafee, T.; Chang, S.; Stephens, R.; Elliot, J.; Oliver, S.; John, D.; Smith, M.; Orr, N. Standardised Data on InitiativesSTARDIT: Alpha Version. OSF Prepr. 2019. [CrossRef]

42. Saldana, J. The Coding Manual for Qualitative Researchers; SAGE Publications: Los Angeles, CA, USA, 2013.

43. Hyett, N.; Kenny, A.; Dickson-Swift, V. Methodology or method? A critical review of qualitative case study reports. Int. J. Qual. Stud. Health Well-Being 2014, 9, 23606. [CrossRef] [PubMed]

44. Michelle Man, T.A. Rosie McLeod. In Implementing and Evaluating Co-Design: A Step-by-Step Toolkit; New Philanthropy Capital: London, UK, 2019. 